\title{
Intelligent and safe design of recycling express packing boxes
}

\author{
Jian Gong ${ }^{1}$, Xingyue $\mathrm{Lu}^{1}$, Jie He ${ }^{1}$, a , Jiajia $\mathrm{Li}^{1}$, Xintong Yan ${ }^{1}$, Hao Zhang ${ }^{1}$ and Changjian Zhang ${ }^{1}$ \\ ${ }^{1}$ School of Transportation, Southeast University, Nanjing 210018, China
}

\begin{abstract}
In order to cope with the growing environmental pollution triggered by traditional disposable express packing box, the design and promotion of recycling express packing boxes (REPB) is of necessity and feasibility. However, relevant research is still lacking. To address the aforementioned issues, this paper attempted to propose a comprehensive method for designing REPB based on five intelligent functions of REPB, i.e., information processing, transportation environmental state perception, transportation environment interaction, human-machine interaction, and cloud interaction. Additionally, to ensure the reliability and safety of REFB, this paper discussed the material and structure of REPB based on shock absorption, as well as the intelligent functions to protect REFB from being stolen and lost. This paper can aid in the establishment of framework for REPB design pertaining to intelligence, safety, feasibility and reliability. Future research could further explore the standardization of REFB.
\end{abstract}

\section{Introduction}

\subsection{Background}

With the rapid development of the e-commerce industry, the number of express delivery orders has also maintained rapid growth. In 2018, China's express delivery business reached 50.71 billion pieces, $26.6 \%$ more than 2017 .

In China, the express bills in 2017 were about 40.06 billion pieces, leading to massive consumption of express package accessories (e.g., packaging bags 11.05 billion, packaging boxes 1.2 billion). In contrast to the large amount of resources consumed, the recovery rate of cardboard and plastic is currently less than $10 \%$ [1], indicating not only the waste of substantial resources wasted but also the generation of extensive pollution. Furthermore, due to the lack of the promotion and application, the cost of recycling and remanufacturing for express package is high. Therefore, the promotion of green packaging in the express delivery industry is of great significance.

In addition, for the sake of low cost, express delivery is mostly disposable packaging (i.e., it can only be used once before it is discarded), relying on paper sheets to contain information, which constrains amelioration of information technology in the logistics process [2].

To address the aforementioned problems, there is a necessity to develop the recycling express packing boxes (REPB), which can be reused and thus reduce waste of resources and environmental contamination. Additionally, more functions can be integrated into the design of REPB for the enhancement of the efficiency, security and reliability of REPB, and subsequently improve the

a Corresponding author: hejie@seu.edu.cn efficiency of the logistics industry.

\subsection{Related work}

From the perspective of intelligent logistics, Zhang [3] (2017) used AHP (Analytic Hierarchy Process) to measure the actual level and economic value of the development of China's intelligent logistics industry. Schaefer and Cheung [4] (2018) specialized in intelligent packaging and analyzed the opportunities and challenges of intelligent packaging.

Based on the application of IoT (Internet of Things) in the logistics domain, Gao [5] (2017) integrated Bluetooth technology with low energy to build a security monitoring system for storage circumstance. Jiang [6] (2017) argued that radio frequency identification (RFID) has a higher recognition speed and accuracy that bar code. Yu et al. [7] (2016) designed a logistics monitoring system based on Beidou navigation and Bluetooth. This system can record the state parameters of the cargo (e.g., acceleration, temperature, humidity and inclination).

With regards to safety and reliability, Wang et al. [8] (2017) studied the material for green packaging. Yao et al. [9] (2013) proposed an intelligent monitoring system for the transportation of dangerous goods combining IoT technologies, with the advantages of rationality, stability and scalability.

It can be found that the current technology of intelligent express packaging boxes is basically mature and has been primarily applied. However, the existing research integrating the notion of intelligence into REPB is still lacking, which deserves further exploration. This paper intends to provide a framework for the design of REPB in terms of intelligence and security. The method 
proposed in this paper can provide support for the design and development of REPB, so that designers can integrate mature technologies more efficiently.

\section{Intelligent design of REPB}

\subsection{Intelligent Function Design}

For adapting to the increasingly complete intelligent logistics system, green express packaging boxes requires intelligent design. Packaging is the underlying intelligent terminal in the intelligent logistics network. The following five types of information interactions are involved in the packaging transportation and recycling process, including information processing, environmental state perception, transportation environment interaction, human-machine interaction, and cloud interaction.

\subsubsection{Information processing}

Information processing is the most basic information operation of intelligent packaging. Intelligent packaging is required to perform certain processing before making a judgment, whether data is collected by sensors or from cloud and other secondary terminals. After the information is processed, operation instructions such as erasing, writing, and sending need to be performed.

This article proposed a " $3+1$ " information processing system, i.e., "3" means the establishment of a three-level automatic processing system (intelligent express packing box - transport environment-cloud); "1" is the manual intervention as an assisting processing means, as shown in Figure 1.

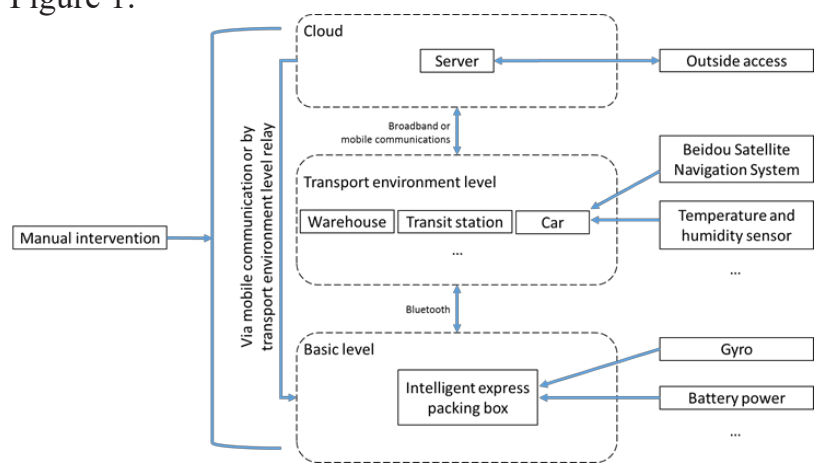

Figure 1. Schematic of the " $3+1 "$ information processing system.

\subsubsection{Environmental state perception}

Environmental state perception refers to the data directly collected by the package through various sensors. The information required for express packaging includes the following: battery status, location and speed, vibration and shock, temperature and humidity, storage direction, and pressure. The environmental state perception solution is shown in Figure 2. Battery status, position and speed, vibration and shock are relatively common data; temperature and humidity, storage direction and pressure data are applicable to fragile and fragile goods.

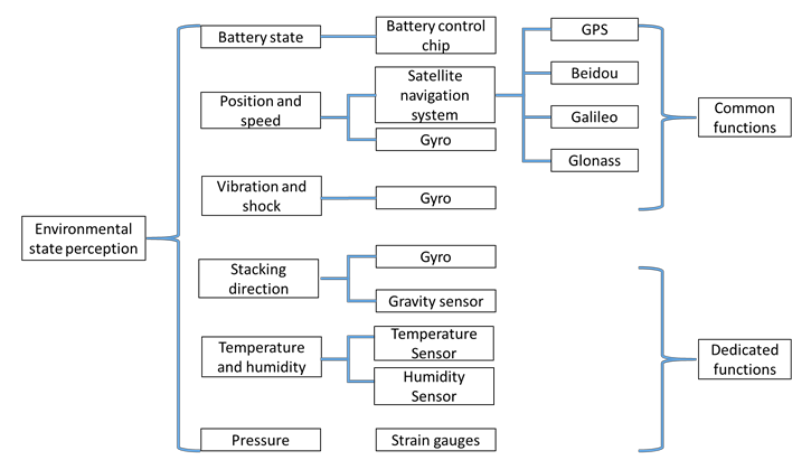

Figure 2. Schematic diagram of environmental state perception solution.

\subsubsection{Transportation environment interaction}

Transportation environment interaction refers to the information interaction between express packaging and higher-level terminals in the transportation environment (shown in Figure 3). For example, an express package stored in a warehouse can exchange information with a terminal in the warehouse. Information interaction with the transportation environment can help achieve multi-source information integration of higher-level terminals, improve the efficiency of information collection as a whole, and reduce hardware costs.

The most widely used technologies are Bluetooth, WLAN, and RFID. Their features can be concluded as follows:

- The intelligent express packaging box is restricted by electric energy. From the perspective of energy consumption, and the use of WLAN technology is excluded;

- For situations where a long-term connection is required to report and receive data at any time, Bluetooth 4.0 technology is recommended;

- For operations such as automatic sorting that require rapid identification and shunting, RFID based on passive cards is recommended.

\subsubsection{Cloud interaction}

Cloud interaction means that the express packaging can access the network and directly interact with the server of the logistics system. It relies on mobile communication technologies represented by $4 \mathrm{G}$ and $5 \mathrm{G}$, and receives instructions from the cloud. This is also the most direct way to interact with information.

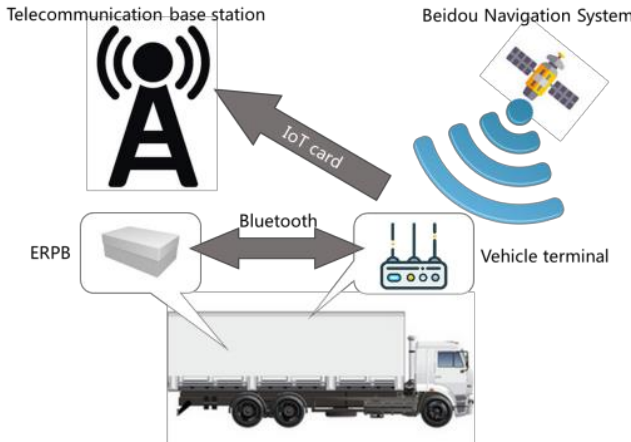

Figure 3. Information interaction of intelligent express boxes. 


\subsubsection{Human-machine interaction}

Human-machine interaction refers to the interaction between a person and a box. Express packaging boxes can use output devices such as display screens and buzzers for feedback.

Human-machine interaction is a one-to-one interaction between the staff and REPB. There are two types of interaction situations. One is to obtain the ID number of the box from the cloud to find out its related information, and the other is to exchange information with the packaging box directly through a handheld terminal.

The box can also use the display, buzzer, and indicator light to output information to people. The REPB can be equipped with a cheap OLED monochrome display screen. The buzzer can issue a warning message or sound under controlled conditions to facilitate searching the REPB. The indicator light can indicate the working status.

The importance of these five functions have been concluded in Table 1 .

Table 1. Necessity of intelligent functions.

\begin{tabular}{|l|l|}
\hline Necessity & Functions \\
\hline Indispensable & $\begin{array}{l}\text { Information processing; } \\
\text { Human-machine interaction }\end{array}$ \\
\hline Replaceable & $\begin{array}{l}\text { Environmental state perception; } \\
\text { Cloud interaction; } \\
\text { Transportation environment interaction; }\end{array}$ \\
\hline
\end{tabular}

\subsection{Analysis of function options}

After discussing intelligent functions and hardware solutions, three optional design schemes were proposed to divide the intelligent express packaging box into basic models, upgrade models and special transport models. The basic and upgraded models are general-purpose REPB. The special transport models are applicable to special requirements of goods, such as important paper documents.

In terms of communication methods, Bluetooth is used to communicate with the transportation environment, and then indirectly communicate with the cloud. In order to improve reliability, the special transport model is equipped with a mobile communication IoT card. The detailed configuration scheme is shown in Table 2.

Table 2. Function matching scheme

is available, $\bigcirc$ is optional, $\times$ is none).

\begin{tabular}{|l|l|l|l|}
\hline Function & $\begin{array}{l}\text { Basic } \\
\text { Model }\end{array}$ & $\begin{array}{l}\text { Upgrade } \\
\text { Model }\end{array}$ & $\begin{array}{l}\text { Special } \\
\text { transport } \\
\text { Model }\end{array}$ \\
\hline GPS/Beidou & $\times$ & $\bigcirc$ & $\bigcirc$ \\
\hline Gyro & $\times$ & $\bigcirc$ & $\bigcirc$ \\
\hline Sensors & $\times$ & $\times$ & $\bigcirc$ \\
\hline 2G/3G/4G/5G & $\times$ & $\bigcirc$ & \\
\hline Bluetooth & & $\bigcirc$ & \\
\hline RF card & $\bigcirc$ & 0 & \\
\hline
\end{tabular}

\begin{tabular}{|l|l|l|l|}
\hline Display & $\mathbf{0}$ & $\mathbf{0}$ & $\mathbf{0}$ \\
\hline Buzzer & $\mathbf{0}$ & $\mathbf{0}$ & $\mathbf{0}$ \\
\hline Indicator light & $\mathbf{0}$ & $\mathbf{0}$ & $\mathbf{0}$ \\
\hline
\end{tabular}

\section{3 security design of REPB}

Security is mainly for the goods in the box. The most important task of the REPB is to ensure that the goods can be delivered to the recipient intact.

\subsection{Shock absorption design}

There are many cases of packages colliding and falling in the process of express delivery. The impact of these complex situations has a large and widespread threat to the safety of goods. The shock absorption design includes the selection of the main material and the design of the internal buffer layer.

\subsubsection{Selection of main materials}

The main material selection of express green packaging boxes includes two levels: one is the raw materials for packaging, and the other is the structure of the materials. Packaging materials include wood, metal, plastic, paper, etc. Considering the application scenarios, REPB usually uses plastic as the raw material, which has a great effect for the shock absorption ${ }^{[10]}$.

\subsubsection{Design of the internal buffer layer}

The design of the buffer layer is usually designed in the form of flexible cushions, integrated airbags and row airbags. The features can be concluded as Table 3 .

Table 3. Features of REPB internal buffer layer.

\begin{tabular}{|l|l|l|}
\hline Form & Advantages & Disadvantages \\
\hline Flexible cushions & Low costs & Low adaptability \\
\hline Integral airbag & $\begin{array}{l}\text { difficult to } \\
\text { be damaged }\end{array}$ & difficult to be folded \\
\hline Row airbag ${ }^{[1]}$ & $\begin{array}{l}\text { High } \\
\text { efficiency }\end{array}$ & $\begin{array}{l}\text { Weak protection for } \\
\text { goods when shocked } \\
\text { drastically }\end{array}$ \\
\hline
\end{tabular}

\subsection{Anti-theft and anti-lost design of REPB}

There are two ways to track lost package. One is to use satellite positioning to find the location of the lost package, and the other is to use DSRC such as Bluetooth to try to contact the REPB and narrow the search.

Express delivery has many links in the process of transportation and faces the risk of man-made malicious unpacking. Express delivery in traditional packaging has little protection against illegal unpacking. As long as the offender avoids the monitoring area and unpacks the box, the goods can be easily stolen without evident clues. Even if the customer finds it afterwards, it is difficult to 
troubleshoot the entire transportation process. Express logistics companies can't rule out hidden dangers in time.

Sensors can be used to identify and record the violent damage. As shown in Table 4, it is designed to use an electronic seal to determine whether the box has been damaged illegally. A gyroscope can also be used to determine whether it is carried violently. Users and express logistics companies can utilize information such as the location and time recorded by the sensors to accurately find out the problem. This approach contributes greatly to the division of responsibilities, law enforcement, and elimination of hidden risk.

Table 4. Anti-theft and anti-lost design.

\begin{tabular}{|l|l|l|}
\hline Scene & $\begin{array}{l}\text { Hardware } \\
\text { support }\end{array}$ & Functions \\
\hline Loss & DSRC, GPS & Positioning \\
\hline Illegal unpacking & Electronic seal & Recording \\
\hline Violent destruction & Gyro & Recording \\
\hline
\end{tabular}

\section{Conclusion}

In order to solve the environmental problems caused by express packaging, the mainstream solution is to design REPB. This paper studied the design of REPB from the perspectives of intelligence and security. The framework in intelligent function design, shock absorption design, anti-theft and anti-lost design can provide reference for designers. In the future, it is necessary to further study the REPB design scheme in terms of standardization. Also, in terms of operating costs, exploring its promotion and popularization is necessary.

\section{Acknowledgments}

This research is supported by the National Natural Science Foundation of China (71874067 \& 51778141), the "Six Industry Talent Peak Project" fund of Jiangsu Province (RJFW-049 \& JNHB-115), the "Green and Blue Project" fund of Jiangsu Province (2017SJB1641), the Philosophy and Social Science fund for colleges in Jiangsu Province (2017SJB1641 \& 2018SJA1649), the Natural science fund for colleges in Jiangsu Province (17KJB58001), the fund of Huaian Vocational College of Information Technology (HXYC2017003), and the Ministry of education of Humanities and Social Science Project (19YJA790070).

\section{References}

1. H.L. Wang, Y.Q. Li, Z.H. Yin, et al, China's Express Delivery Green Packaging Development Status and Trend Report (Post Bureau, Beijing, China, 2018)

2. X.Y. Lu, Design and Application of Intelligent Shared Express Packaging Box (Southeast University, Nanjing, China, 2019)

3. J.C. Zhang, Evaluation of China's Intelligent Logistics Industry Development Level and Economic Value Analysis (Shanxi University of Finance and Economics, Shanxi, China, 2017)
4. D. Schaefer, W. M. Cheung, Intelligent Packaging: Opportunities and Challenges, Procedia CIRP, 72, 1022-1027 (2018)

5. Y. Gao, Design of remote security monitoring system for storage environment based on Internet of Things (Harbin University of Science and Technology, Harbin, China, 2017)

6. W, Jiang, Research on rapid identification technology for shipbuilding pallet management (Jiangsu University of Science and Technology, Nanjing, China, 2017)

7. Q.J Yu, F.Y Liang, J. Pan, L.X. Gu, G. He, Design of logistics monitoring system based on Beidou and Bluetooth, Measurement \& Control Technology, 35 (01), 50-52 (2016)

8. L. Wang, R.E. Lee, G. Wang, et al, Use of stereocomplex crystallites for fully-biobased microcellular low-density poly (lactic acid) foams for green packaging, Chemical Engineering Journal, 327, 1151-1162 (2017)

9. Z.Q Yao, J. Wang, Y.X Hu, Q.Q Cheng, Y. Qin, RFID /GPRS/GPS/GIS-based Intelligent Supervision System for Dangerous Goods Logistics, Journal of Highway and Transportation Research and Development, 30 (02), 147-152+158 (2013)

10. G.X Peng. Packaging design for logistics and transportation. (Printing Industry Press, Beijing, China, 2006)

11. M. Chen. Study on the mechanical behavior of films and cushioning properties of air cushions. (Shanghai Jiaotong University, Shanghai, China, 2014) 